\title{
Scaling Behavior and Beyond Equilibrium in the Hexagonal Manganites
}

\author{
S. M. Griffin, ${ }^{1}$ M. Lilienblum, ${ }^{1}$ K. T. Delaney, ${ }^{2}$ Y. Kumagai, ${ }^{1}$ M. Fiebig, ${ }^{1}$ and N. A. Spaldin ${ }^{1}$ \\ ${ }^{1}$ Department of Materials, ETH Zurich, Wolfgang-Pauli-Strasse 10, CH-8093 Zurich, Switzerland \\ ${ }^{2}$ Materials Research Laboratory, University of California, Santa Barbara, California 93106, USA
}

(Received 23 July 2012; published 27 December 2012)

\begin{abstract}
We show that the improper ferroelectric phase transition in the multiferroic hexagonal manganites displays appropriate symmetry-breaking characteristics for testing the Kibble-Zurek mechanism originally proposed to describe early-universe phase transitions. We present an analysis of the Kibble-Zurek theory of topological defect formation applied to the hexagonal manganites, discuss the conditions determining the range of cooling rates in which Kibble-Zurek behavior is expected, and show that recent literature data are consistent with our predictions. Finally, we explore experimentally the crossover out of the Kibble-Zurek regime and find a surprising reversal of the scaling behavior.
\end{abstract}

DOI: 10.1103/PhysRevX.2.041022 Subject Areas: Cosmology, Materials Science, Strongly Correlated Materials

\section{INTRODUCTION}

The formation of topological defects such as cosmic strings during phase transitions in the early universe [1-5] was proposed by Kibble [6], who derived the symmetry requirements for their formation. In systems where such topological defects are allowed by the symmetry requirements, the defect density can be estimated using the Zurek mechanism [7], which uses causality arguments to develop scaling laws for the density of defects formed as a function of the rate of quenching across the phase transition. The resulting combination of symmetry requirements and scaling laws is termed the Kibble-Zurek mechanism and in principle should describe a phase transition in any system with the required symmetry properties, provided that other effects do not dominate the kinetics of topological-defect formation.

Attempts to demonstrate Kibble-Zurek scaling in condensed-matter systems have proved challenging, however, and the "ideal Kibble-Zurek system" has previously remained elusive. Zurek's original paper [7] discussed the analogue between cosmic strings and the vortex cores formed in a quench-induced phase transition from normalstate to superfluid ${ }^{4} \mathrm{He}$. However, the corresponding experiment $[8,9]$ yielded large deviations from the predicted behavior, probably because of thermal effects [10]. In ${ }^{3} \mathrm{He}$, the symmetry breaking is closer to that postulated for the early universe $[11,12]$, but the density of topological defects can only be inferred indirectly, and many assumptions must be made to compare with predictions. In superconducting $\mathrm{Nb}$ rings, the density of vortex cores in the superconducting current led to a different scaling exponent than that predicted by the Kibble-Zurek mechanismAgain, experimental artifacts were held responsible [13].

Published by the American Physical Society under the terms of the Creative Commons Attribution 3.0 License. Further distribution of this work must maintain attribution to the author(s) and the published article's title, journal citation, and DOI.
Bose-Einstein condensates could in principle provide a suitable system but are so far subject to experimental limitations [14]. Perhaps the most promising candidates to date are high- $T_{\mathrm{c}}$ superconductors and liquid crystals. In high- $T_{\mathrm{c}}$ superconductors, scaling has been demonstrated and some aspects of the Kibble-Zurek mechanism confirmed, although with large uncertainties [15]. And in liquid crystals, successful studies of defect dynamics have been performed [16], and the diffraction-pattern formation in nonlinear optic experiments has been shown to exhibit a power-law scaling [17]. Strong interactions between the defects continue to cause difficulties, however.

Here we propose the multiferroic hexagonal magnanites, $R \mathrm{MnO}_{3}(R=\mathrm{Sc}, \mathrm{Y}$, Dy to $\mathrm{Lu})$ as a model system for testing the Kibble-Zurek mechanism. The hexagonal manganites have attracted interest because of their unusual geometrically driven improper ferroelectricity, which allows for the simultaneous occurrence of magnetic ordering $[18,19]$, as well as unusual couplings [20-22] and functionalities [23] at their domain walls. In this work we show that the unusual nature of the improper geometric ferroelectric phase transition also sets both the correct symmetry conditions for Kibble-Zurek behavior, as well as the physical properties for readily detecting that behavior. In addition, the relevant time, temperature, and length scales fall into a range that allows exploration of the KibbleZurek regime, as well as the crossover out of it.

\section{SYMMETRY AND PHYSICAL PROPERTIES OF $\mathrm{RMnO}_{3}$}

First, we describe the properties of $R \mathrm{MnO}_{3}$ that are relevant for testing the Kibble-Zurek mechanism, particularly the symmetry properties of the phase transition. The structure of $R \mathrm{MnO}_{3}$ consists of planes of $\mathrm{MnO}_{5}$ trigonal bipyramids separated by planes of $R$ ions which form a hexagonal mesh [Fig. 1(a)] [24]. In the high-temperature paraelectric phase, the space group is centrosymmetric $\mathrm{P}_{3} / \mathrm{mmc}$. At the Curie temperature $T_{\mathrm{C}} \sim 1400 \mathrm{~K}$ (the 

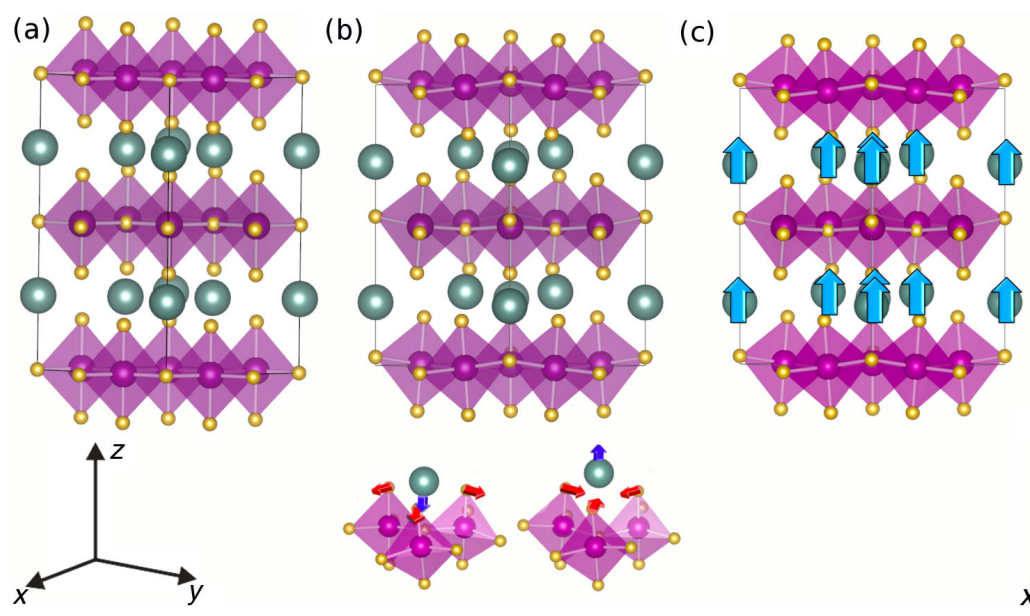

(d)
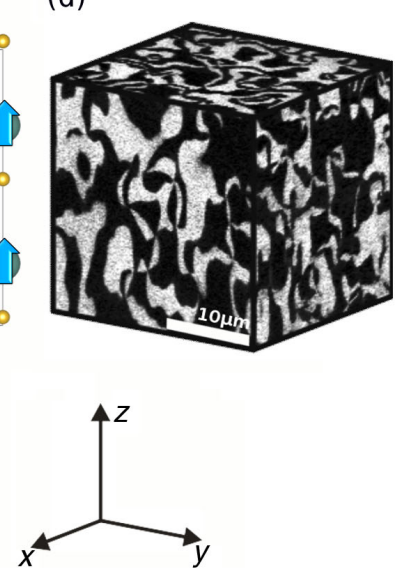

FIG. 1. (a) High-symmetry $P 6_{3} / m m c$ structure of $R \mathrm{MnO}_{3}$ before the onset of trimerization. (b) Action of the $K_{3}$ trimerization mode on the $R$ ions and $\mathrm{MnO}_{5}$ trigonal bipyramids. The insets below the image of the main structure emphasize that outward trimerization results in a downward shift of the corresponding $R$ ion, whereas inward trimerization results in an upward shift. (c) The subsequent additional displacements of the $R$ ions (blue arrows) in the $\Gamma_{2}^{-}$mode provide the ferroelectricity. Note that, once the orientation of the trimerization mode is set, the spontaneous polarization can emerge in only one direction. (d) Typical domain structure measured using piezoresponse force microscopy. The black and white regions correspond to opposite orientations of the ferroelectric polarization along the $z$ axis. Note that the domain structure is isotropic, in spite of the layered crystal structure.

exact value depends on the $R$ ion), a spontaneous symmetry breaking occurs, with the condensation of primarily two phonon modes with distinct irreducible representations of the high-symmetry structure [19,25]. The first is a mode of $K_{3}$ symmetry, which involves a trimerizing tilt of the trigonal bipyramids and is the primary-order parameter [Fig. 1(b)]. Since the $K_{3}$ mode can condense about three different origins, and the tilt can be in the "in" or "out" direction, six trimerization domains are formed; these have been shown using high-resolution transmission electron microscopy to meet at vortex cores [22]. Importantly (and unusually), while this mode lowers the symmetry to that of a polar space group, it carries no net polarization, as any net local polarity vanishes macroscopically due to the nonzero-mode wave vector. A secondary mode of $\Gamma_{2}^{-}$ symmetry (referring to the parent space group), which does not further lower the symmetry, provides the ferroelectric polarization [Fig. 1(c)]. The orientation of this secondary ferroelectric polarization is set by the in or out tilt of the $K_{3}$ mode, and so it does not result in additional domains. It is essential for our experiments, however, as it allows the straightforward imaging of the domain structure using piezoresponse force microscopy (PFM). Indeed, PFM measurements reveal that domains of alternating polarization are locked to the trimerization domains around vortex cores [22,26], yielding appealing sixfold patterns [Fig. 1(d)]. Electric-field poling experiments have shown that the vortex cores are protected in the sense that they cannot be annihilated or driven out of the system by an electric field $[22,26]$. Surprisingly, the domain structure and density of these topological defects when viewed from the side of the sample are similar to those characteristics viewed from the top in spite of the layered crystal structure and uniaxial ferroelectricity [26] [Fig. 1(d)]. This absence of anisotropy in the domain structure allows for straightforward determination of the defect densities from twodimensional top-view scans of their areal density, rather than requiring a complex three-dimensional analysis.

First-principles calculations [19] and Landau-theory analysis [27] have shown that, for small magnitudes of the trimerizing $K_{3}$ mode, the polar mode appears only as a third-order term, and so the magnitude of the ferroelectric polarization just below $T_{\mathrm{C}}$ is vanishingly small. The vanishingly small ferroelectric polarization is important for our discussion for two reasons: First, the formation of the domain structure at $T_{\mathrm{C}}$ is not influenced by the system's attempts to minimize the depolarizing field from the ferroelectric polarization. Strong evidence for this lack of influence is given by the large numbers of electrostatically unfavorable head-to-head and tail-to-tail domain walls that form in $R \mathrm{MnO}_{3}$ but that rarely occur in conventional ferroelectrics [23]. Second, first-principles calculations show that the energy lowering provided by the condensation of the $K_{3}$ mode is independent of the angle of the tilt until the polar mode subsequently develops [27]. This observation means that the potential below the phase-transition temperature is given by the continuous "Mexican-hat" form (Fig. 2). The atomic nature of the lattice does not manifest itself until at lower temperatures when the domain structure is already determined. As a result, we can use the mathematics of continuous symmetries, which are usually assumed in the Kibble-Zurek mechanism and are a condition for the discussion of topological defects. In this language, the full rotational symmetry is broken when the 


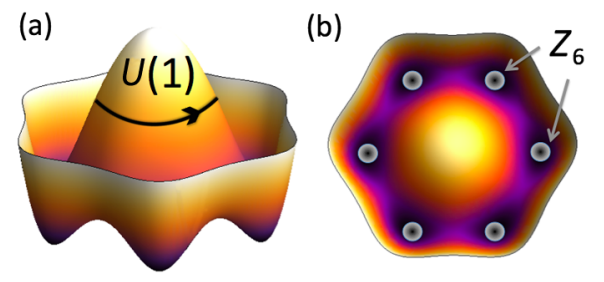

FIG. 2. Mexican-hat potential-energy surface of the hexagonal manganites. At high energy (the peak of the hat), the energy is independent of the angle of trimerization, and the system has continuous $U(1)$ symmetry. At lower energy (in the brim of the hat), six of the trimerization angles become favorable (white circles), and the symmetry reduces to the sixfold discrete symmetry described by $Z_{6}$.

polyhedrons tilt in the $2 \pi$ range of angles, resulting in a $U(1)$ vacuum. We note also that the Landau free energy derived in Ref. [27] already gives a signature of topological protection as a $2 \pi$ rotation of the trimerization angle around the vortex core crosses a branch cut in the free energy.

For larger magnitudes of the $K_{3}$ mode, obtained on temperature decrease, a crossover to linear coupling with the polar $\Gamma_{2}^{-}$mode occurs, and the polarization becomes measurably large. This result lifts the degeneracy of the angle of the $K_{3}$ mode and fixes the polyhedrons into discrete tilt angles of $0,2 \pi / 3$, or $4 \pi / 3$, described by $Z_{3}$ symmetry. The additional degeneracy provided by the direction (in or out) of the polyhedral tilting gives an additional $Z_{2}$ symmetry reduction, resulting in $Z_{2} \times Z_{3}=$ $Z_{6}$. It is an open experimental question whether the onset of the $\Gamma_{2}^{-}$mode, which is observed approximately $300 \mathrm{~K}$ below $T_{\mathrm{C}}$ is an "emergence" or an additional isosymmetric phase transition $[19,25]$.

\section{KIBBLE-ZUREK MECHANISM FOR $\mathrm{RMnO}_{3}$}

In this section, we first show that the symmetry of $R \mathrm{MnO}_{3}$ results in topologically protected vortex cores as described by the Kibble mechanism. We then analyze the vortex cores using the Zurek mechanism to determine the density of topological defects that should be produced as a function of the cooling rate through the phase transition. We use first-principles density-functional theory to evaluate the relevant parameters, and show that our predictions are in agreement with literature data.

\section{A. Kibble mechanism and the formation of topological defects}

The requirements for the formation of topological defects at a phase transition within the Kibble mechanism [6] are (i) a spontaneous symmetry breaking and (ii) a change in symmetry across the phase transition that corresponds to a nontrivial homotopy group. The trimerization transition in $R \mathrm{MnO}_{3}$ clearly fulfills the first condition; next, we show that it also fulfills the second.

As discussed earlier, in the temperature range just below the phase transition, $R \mathrm{MnO}_{3}$ exhibits a continuous symmetry. This fact allows us to use the methods and results of homotopy theory-which have been developed for continuous-symmetry groups - to assess the topology of $R \mathrm{MnO}_{3}$. It is established within homotopy theory that the symmetry characteristics of the order parameter, in our case, $U(1)$, can be used to assess the topological characteristics of a phase transition. To make the assessment, the order-parameter symmetry is first mapped onto an $n$-dimensional sphere. In the case of $U(1)$ symmetry, this map is a one-dimensional circle, $S^{1}$. Next, we define a function called the homotopy group, $\pi_{k}$, which describes the topological nature of the order-parameter symmetry. If $\pi_{k}$ differs from the identity, then it is nontrivial and topological defects are formed. It has been known since the 1960s [28] that $\pi_{k}\left(S^{1}\right)$ is indeed nontrivial and in fact produces one-dimensional topological singularities, called strings or vortex cores [29]. Therefore, the vortex cores in $R \mathrm{MnO}_{3}$ are mathematically topologically protected, in concordance with their physical topological protectiontheir resistance to annihilation by an electric field - that we discussed earlier [22,26]. We also note that within the Kibble mechanism the topological defects are remnants of the parent phase trapped within the lower symmetry phase. For $R \mathrm{MnO}_{3}$, this observation implies that the highsymmetry paraelectric phase is preserved at the meeting point of the six domains defining a vortex core.

\section{B. Zurek mechanism for $\mathrm{RMnO}_{3}$}

Within the Zurek mechanism, the density of topological defects formed during a spontaneous symmetry-breaking phase transition described by the Kibble mechanism follows a power-law dependence on the rate at which the transition is crossed [7,30]. In this section, we first relate the material properties of $R \mathrm{MnO}_{3}$ to the parameters in the Zurek mechanism. We then evaluate their magnitudes to calculate quantitatively the temperature dependence of the defect formation within the Kibble-Zurek mechanism.

Zurek's approach relies on the notion of competing time scales: The first relevant time scale is the time it takes for one region of the system to communicate its choice of vacuum state with another. This scale sets a "sonic horizon" within which the order parameter chooses the same vacuum state. This communication time becomes divergently long as the critical temperature is approached and the correlation length diverges, a phenomenon termed "critical slowing down." The second relevant time scale is the quench time $\tau_{q}$ that the system spends cooling through the phase transition. The size of the domains is set at the temperature $T_{\mathrm{f}}=T_{\mathrm{C}}+\Delta T_{\mathrm{f}}$ where the communication distance across which information can be transferred during the progressing phase transition becomes equal to the correlation length $\xi(T)$ (Fig. 3). As the temperature further approaches $T_{\mathrm{C}}$, the correlation length $\xi(T)$ continues to diverge but the communication length remains unchanged, and the system is unable to adapt to the 


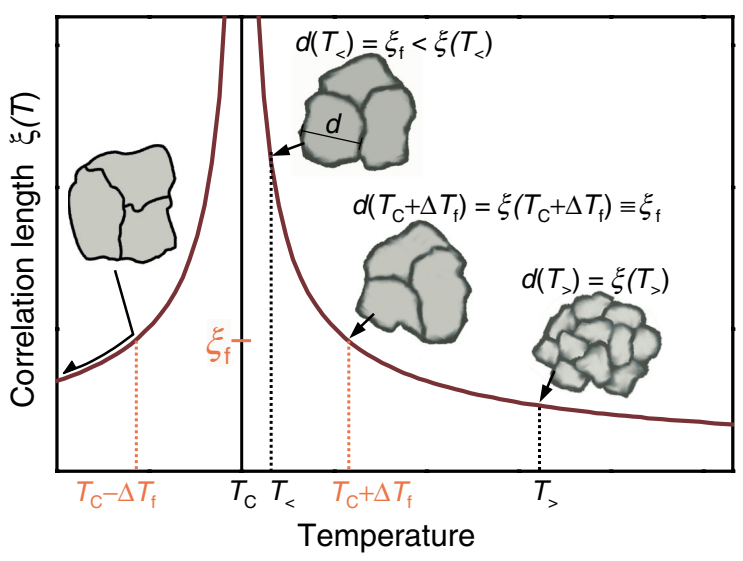

FIG. 3. Domain formation and the Kibble-Zurek mechanism. Above $T_{\mathrm{C}}$, fluctuating regions of lateral extension $d$ occur with uniform orientation of the emerging order parameter (fuzzy patches). At high temperature $\left(T_{>}>T_{\mathrm{C}}+\Delta T_{\mathrm{f}}\right)$, the size of the correlated regions is determined by the correlation length (purple curve). At temperature $T_{\mathrm{f}}=T_{\mathrm{C}}+\Delta T_{\mathrm{f}}$, a freeze-out of the lateral extension $d$ begins, and below the freeze-out temperature, the lateral extension of the fluctuating regions can no longer match the diverging correlation length. The size of the fluctuating regions at temperatures $T_{<}<T_{\mathrm{C}}+\Delta T_{\mathrm{f}}$ is set by the correlation length at the freeze-out temperature, $\xi_{f}=$ $\xi\left(T_{\mathrm{C}}+\Delta T_{\mathrm{f}}\right)$, and corresponds to the "communication length," which is the distance that information propagates during the time in which the system cools from $T_{\mathrm{C}}+\Delta T_{\mathrm{f}}$ to $T_{\mathrm{C}}-\Delta T_{\mathrm{f}}$ (red vertical lines). Below $T_{\mathrm{C}}-\Delta T_{\mathrm{f}}$, stable domains of lateral extension $\xi\left(T_{\mathrm{C}}+\Delta T_{\mathrm{f}}\right)$ form (indicated by solid black domain boundaries).

increase in correlation length. As a consequence a "freeze-out" occurs in the temperature interval between $T_{\mathrm{C}}+\Delta T_{\mathrm{f}}$ and $T_{\mathrm{C}}-\Delta T_{\mathrm{f}}$ : The size of the correlated regions is unable to increase and so the domain size is fixed at the value $\xi\left(T_{\mathrm{C}}+\Delta T_{\mathrm{f}}\right)$ (Fig. 3). For fast cooling through the transition, the distance over which information can be transferred during the transition is small, and becomes equal to the correlation length at small values of $\xi(T)$. Therefore, freeze-out occurs when the domain size is small (and consequently the number of topological defects is large). In contrast, for slow cooling, the distance for information transfer is large, and does not become equal to $\xi(T)$ until close to the phase transition temperature, where $\xi(T)$ is large. In this case, large domains, with fewer topological defects, form.

Here, we summarize the derivation of the density of topological defects as a function of quench rate through the phase transition within the Kibble-Zurek mechanism. For a detailed derivation, we recommend particularly Ref. [31]. First, we use critical scaling: As the system approaches $T_{\mathrm{C}}$, the correlation length, $\xi$, and relaxation time, $\tau$, diverge as

$$
\xi(T)=\xi_{0}\left|1-\frac{T}{T_{\mathrm{C}}}\right|^{-\nu}, \quad \tau(T)=\tau_{0}\left|1-\frac{T}{T_{\mathrm{C}}}\right|^{-\mu},
$$

where $\xi_{0}$ is the zero-temperature correlation length and $\tau_{0}$ is the zero-temperature time, which is equal to $\xi_{0}$ divided by the speed of information transfer in the system. Both $\xi_{0}$ and $\tau_{0}$ are system-dependent quantities. $\nu$ and $\mu$ are critical exponents that are determined by the universality class of the phase transition, that is, its general behavior as determined by the symmetry properties of the phase transition, irrespective of the material properties of the specific system.

Assuming that the temperature varies linearly with time near the phase transition, and taking $t=0$ at $T=T_{\mathrm{C}}$, it is clear that $T=T_{\mathrm{C}}-\left(t \times r_{\mathrm{q}}\right)$, where $r_{\mathrm{q}}$ is the cooling rate. Rearranging this expression yields

$$
\left|1-\frac{T}{T_{\mathrm{C}}}\right|=\frac{r_{\mathrm{q}}}{T_{\mathrm{C}}} t=\frac{t}{\tau_{\mathrm{q}}}
$$

where $\tau_{\mathrm{q}}=\frac{T_{\mathrm{C}}}{r_{\mathrm{q}}}$ is called the "quench" time.

The speed at which information is transferred in the material is then given by the characteristic velocity,

$$
c(T)=\frac{\xi(T)}{\tau(T)}=\frac{\xi_{0}}{\tau_{0}}\left|1-\frac{T}{T_{\mathrm{C}}}\right|^{\mu-\nu},
$$

and the corresponding distance over which information can propagate in time $t$ is

$$
\begin{aligned}
\int_{0}^{t} c\left[T\left(t^{\prime}\right)\right] d t^{\prime} & =\frac{\xi_{0}}{\tau_{0}} \int_{0}^{t}\left(\frac{t^{\prime}}{\tau_{\mathrm{q}}}\right)^{\mu-\nu} d t^{\prime} \\
& =\frac{1}{1+\mu-\nu} \frac{\xi_{0}}{\tau_{0}} \tau_{\mathrm{q}}\left|1-\frac{T}{T_{\mathrm{C}}}\right|^{1+\mu-\nu},
\end{aligned}
$$

where in the last step we have substituted $t=\left|1-\frac{T}{T_{\mathrm{C}}}\right| \tau_{q}$.

Equating the distance over which information can propagate to the correlation length yields an expression for the freeze-in temperature, $T_{\mathrm{f}}$,

$$
\frac{1}{1+\mu-\nu} \frac{\xi_{0}}{\tau_{0}} \tau_{\mathrm{q}}\left|1-\frac{T_{\mathrm{f}}}{T_{\mathrm{C}}}\right|^{1+\mu-\nu}=\xi_{0}\left|1-\frac{T_{\mathrm{f}}}{T_{\mathrm{C}}}\right|^{-\nu},
$$

so

$$
\left|1-\frac{T_{\mathrm{f}}}{T_{\mathrm{C}}}\right|=\left((1+\mu-\nu) \frac{\tau_{0}}{\tau_{\mathrm{q}}}\right)^{1 /(1+\mu)} .
$$

At temperature $T_{\mathrm{f}}$, the domain sizes are frozen in with a characteristic length scale given by the informationpropagation distance correlation at the freeze-in temperature:

$$
\xi_{\mathrm{f}}=\xi_{0}(1+\mu-\nu)^{[-\nu /(1+\mu)]}\left(\frac{\tau_{\mathrm{q}}}{\tau_{0}}\right)^{(\nu / 1+\mu)} .
$$

Vortex strings then form at the intersections of these domains with a density of around one $\xi_{\mathrm{f}}$ of length in a volume of $\xi_{\mathrm{f}}^{3}$. The number of vortex intersections per unit area, $n$, is then approximately equal to the length of vortex strings per unit volume, $\frac{1}{\xi_{\mathrm{f}}^{2}}$, giving 


$$
n \approx \frac{1}{\xi_{0}^{2}}\left(\frac{\tau_{0}}{\tau_{\mathrm{q}}}\right)^{(2 \nu / 1+\mu)}
$$

To apply the scaling law that we have derived above to the hexagonal manganites, we next identify the relevant time and length scales in the system, and evaluate their magnitudes. Our electronic-structure calculations have been performed using density-functional theory within the local density + Hubbard $U$ approximation following the Liechtenstein approach [32] with the double-counting corrections treated in the fully localized limit. Following previous literature studies [19], we set the parameters of local density approximation + Hubbard $U(\mathrm{LDA}+U)$ on the Mn $3 d$ orbitals to $U=8$ and $J=0.88 \mathrm{eV}$, respectively, and enforce an A-type antiferromagnetic ordering. We use the projector-augmented wave method for corevalence partitioning [33], which significantly reduces the required plane-wave energy cutoff, and have carefully tested the convergence of plane-wave-cutoff and k-point sampling.

The zero-temperature correlation length, $\xi_{0}$, is usually equated with the zero-temperature domain-wall width in ferroelectrics. In order to extract this value, we perform density-functional calculations within the $\mathrm{LDA}+U$ method using the VASP code [34,35]. We construct supercells containing two $180^{\circ}$ domain walls and, in turn, 120 , 180,240 , and 300 atoms. We initialize a different trimerization phase and ferroelectric orientation within adjacent domains, and fix the lattice constants of the supercells to those of the corresponding relaxed single-domain supercells. We then perform full relaxations on the structures, optimizing the internal positions until the forces acting on all atoms converge to less than $0.01 \mathrm{eV} / \AA$, respectively; in all cases, the system remains in the metastable multidomain state. For all supercell sizes, we have found that the structural phase defined by either the tilt of the $\mathrm{MnO}$ bipyramids or the direction of off-centering of the $\mathrm{Y}$ ions changes abruptly at the domain walls, indicating an effective domain-wall width close to zero. This finding sets an upper limit on $\xi_{0}$ of the interatomic spacing of approximately $1 \AA$. While unusually narrow for a ferroelectric domain wall, such abrupt walls are not atypical for antiphase boundaries, and indeed our calculated value is consistent with a recent experimental electron-microscopy study at room temperature [36], indicating that our calculated zero-kelvin value is relevant over a wide temperature range. As an additional check, we have repeated our calculations for walls between domains of different trimerization phase and the same ferroelectric orientation, as well as between domains of opposite polarity but the same phase, and in all cases have obtained abrupt boundaries [37].

To calculate the characteristic time scale of the system, $\tau_{0}=\frac{\xi_{0}}{s}$, we require $s$, which is the speed at which the system communicates the lattice distortion as it passes through the phase transition. For structural phase transitions in solid-state systems, $s$ is given by the relevant speed of sound. To calculate the speed of sound at zero kelvin, we use the ABINIT [38] software package $[39,40]$ to optimize the structure of a 10-atom unit cell and then calculate the full phonon band structure using frozenphonon techniques. We constructed a supercell with doubling and trebling in each of the directions required to sample the first Brillouin zone, and then we made symmetry-distinct displacements to construct the full matrix of interatomic force constants. The dynamical matrix is diagonalized along each of the high-symmetry lines shown in the phonon band structure using Fourier interpolation $[41,42]$. We then extract the speed of sound from the calculated phonon band structure by fitting the acoustic branch with a polynomial, and then we evaluate the group velocity:

$$
v_{\mathrm{g}}=\left.\frac{\partial \omega}{\partial \vec{k}}\right|_{\vec{k}=0} .
$$

Because we analyze the vortex density in the $a b$ plane as a function of quench rate, the relevant velocity for our Kibble-Zurek fit is the doubly degenerate branch with the atoms displacing in plane and the wave vector propagating in plane. For this branch, we obtain $v_{\mathrm{g}}=640 \mathrm{~m} \mathrm{~s}^{-1}$. We note that the measured lattice constants change only a small amount between low temperature and the phasetransition temperature, indicating that beyond-harmonic lattice-dynamic effects are unimportant, and the calculated zero-kelvin value is also relevant for the scaling regime.

For comparison with quenching experiments, we also need the Curie temperature, $T_{\mathrm{C}}$, which relates $\tau_{\mathrm{q}}$ to the cooling rate, $r_{\mathrm{q}}$ through $r_{\mathrm{q}}=\frac{T_{\mathrm{C}}}{\tau_{\mathrm{Q}}}$. This value is known experimentally to be about $1400 \mathrm{~K}$, with the exact number depending on the $R$ ion.

Finally, we extract the critical exponents by identifying that the $R \mathrm{MnO}_{3}$ transition belongs to the universality class of the 3D XY model. Here, we use the result of Ref. [27], which is that, before the emergence of the polarization, the trimerization has full XY symmetry, with a 3D order parameter that can be modulated in all three spatial dimensions. (2D XY behavior can also be discounted: first, because the 2D XY model does not give a phase transition, and second, because our studies of the side faces of $\mathrm{YMnO}_{3}$ samples show the same domain-formation patterns as the top faces [23].) The values of the critical exponents for the 3D XY universality class have been calculated using Monte Carlo simulations [43] to be $\nu=$ 0.6717 and $\mu=1.3132$, giving a Kibble-Zurek scaling exponent of $\frac{\nu}{1+\mu} \approx 0.29$. This value is very close to the experimental value of 0.27 , found by best fitting to the K3mode order parameter [44]. Taking the values introduced so far, with our upper limit for $\xi_{0}$, we find that domains of $5-\mu \mathrm{m}$ width should be formed for a quench time of $\tau_{\mathrm{q}} \sim$ $40 \mathrm{~min}$ (corresponding to a cooling rate of about $0.5 \mathrm{~K} / \mathrm{s}$ ), 

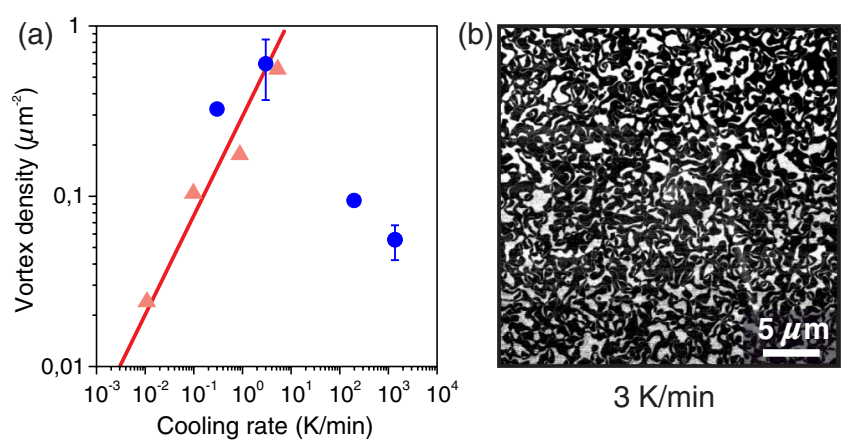

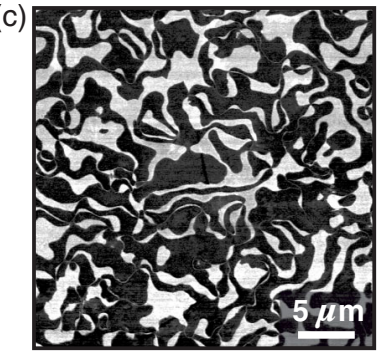

$195 \mathrm{~K} / \mathrm{min}$

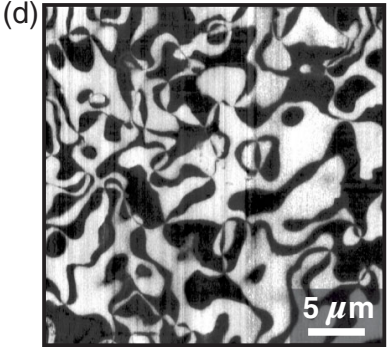

$1360 \mathrm{~K} / \mathrm{min}$

FIG. 4. (a) Areal vortex-core density as a function of cooling rate for slow cooling (red triangles, Ref. [45]) and fast cooling (blue circles, this work). Note the turnover in the cooling-rate dependence of the vortex-core density occurring at around $10 \mathrm{~K} / \mathrm{min}$. The red solid line is the result of our $a b$ initio application of the Kibble-Zurek mechanism with parameters from first-principles calculations. (b)-(d) Distribution of ferroelectric domains in z-oriented $\mathrm{YMnO}_{3}$ samples after annealing cycles at different cooling rates. The images, which are obtained using PFM on an area $30 \times 30 \mu \mathrm{m}^{2}$, reveal a striking "anti-Kibble-Zurek" behavior, with higher cooling rates leading to larger domains.

and domains of $40 \mu \mathrm{m}$ for a quench time of around one month (cooling rate approximately $1.5 \mathrm{~K} /$ hour). These cooling rates are readily accessible experimentally.

In Fig. 4(a), we compare our calculated scaling behavior with recently reported vortex densities measured as a function of cooling rate in $\mathrm{ErMnO}_{3}$ (red triangles) [45]. We obtain the best match with a value of zero-temperature correlation length of $0.06 \AA$ (red line), consistent with the approximately zero domain-wall width obtained in our density-functional calculations. The agreement in scaling behavior between the experiment and the Kibble-Zurek prediction is clear, with the scaling exponent, in particular, matching the theoretical prediction well.

We thus find a unique situation in $R \mathrm{MnO}_{3}$. Topologically, it is a model system for the experimental verification of the Kibble-Zurek mechanism. In the temperature range where the Kibble-Zurek mechanism is expected to govern the formation of domains and the distribution of vortex-core singularities, the unwanted ferroelectric polarization that could influence domain formation is effectively absent. However, at room temperature, the coupling of the distortive order parameter to the now finite ferroelectric polarization allows straightforward imaging of the topology and vortices via spatially resolved measurements of the ferroelectric domain structure. And finally, we obtain a distinguishable range of domain sizes and hence defect densities for an experimentally accessible range of cooling rates through the primary distortive phase transition at $T_{\mathrm{C}}$.

\section{BEYOND THE KIBBLE-ZUREK LIMIT}

The Kibble-Zurek mechanism applies only to the regime in which the system has time to respond adiabatically to the cooling until the freeze-out temperature, $T_{\mathrm{C}}+\Delta T_{\mathrm{f}}$ is reached. For faster quenching, it is expected that the Kibble-Zurek mechanism should break down and be replaced by a dynamics that is largely unknown [46-49].
Therefore, in the final part of this work, we perform quenching experiments at rates more rapid than those explored in Ref. [45] to investigate whether Kibble-Zurek behavior continues, or whether an evolution out of the Kibble-Zurek regime occurs.

For our experiment, we choose $\mathrm{YMnO}_{3}$ rather than the $\mathrm{ErMnO}_{3}$ that was used in Ref. [45] because of the greater thickness of our $\mathrm{YMnO}_{3}$ samples. The experimental procedure applied to the flux-grown, c-oriented single crystal $\mathrm{YMnO}_{3}$ platelets is shown in Fig. 5. For our annealing experiments, we use a conventional chamber furnace, which allows for temperatures from room temperature up

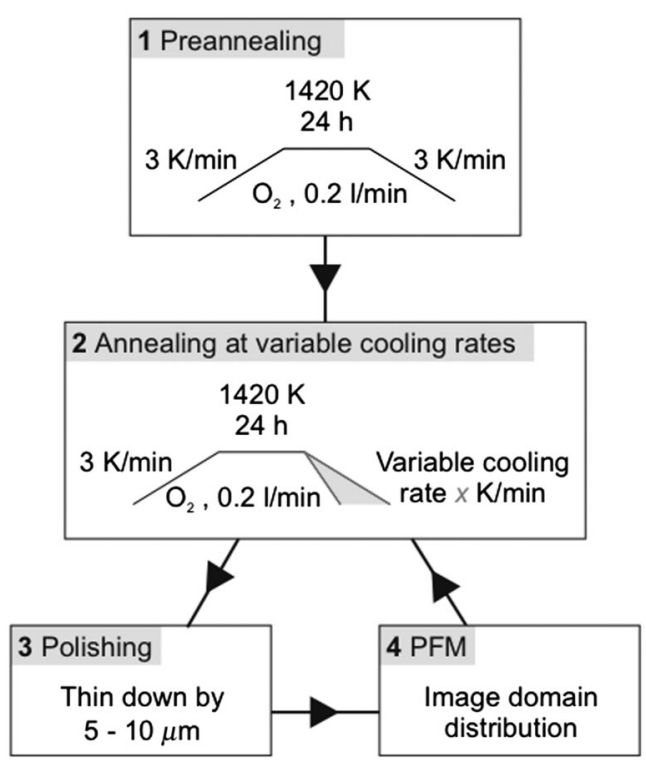

FIG. 5. Sketch of the annealing procedure. After preannealing, the samples are heated to $1420 \mathrm{~K}$ with a hold time of $24 \mathrm{~h}$ and subsequent cooling at rates between 0.1 and $1360 \mathrm{~K} / \mathrm{min}$. After the annealing cycle, the samples are thinned by $5-10 \mu \mathrm{m}$ by polishing in order to access the domain structure formed in the true three-dimensional bulk of the sample. 
to $1550 \mathrm{~K}$, exceeding considerably the $T_{\mathrm{C}} \approx 1270 \mathrm{~K}$ of our $\mathrm{YMnO}_{3}$ samples. First, we perform a preannealing at $1420 \mathrm{~K}$ for $24 \mathrm{~h}$ under constant oxygen flow of $0.2 \mathrm{l} / \mathrm{min}$. Second, the samples are annealed again with a different cooling rate. Up to $8 \mathrm{~K} / \mathrm{min}$, the temperature gradient is controlled by the furnace; higher rates are obtained by removing the fused-silica cell with the sample from the furnace and are measured by an infrared camera. Since the domain structure and density of defects at the surface could in principle be different from those properties in the bulk, we have thinned each sample by 5-10 $\mu \mathrm{m}$ after annealing, using $\mathrm{Al}_{2} \mathrm{O}_{3}$ and polishing with a chemical-mechanical $\mathrm{SiO}_{2}$ slurry with $32-\mathrm{nm}$ grain size. Last, we image the ferroelectric domain structure using PFM $[26,45]$. We use a commercial scanning-force microscope (NTEGRA Solaris, NT-MDT) operating in contact mode and apply an $\mathrm{AC}$-voltage of $14 \mathrm{~V}_{\mathrm{pp}}$ at a frequency of about $40 \mathrm{kHz}$ to a conductive Pt-Ir-coated probe (NSC35, Mikromasch). We record the out-of-plane component of the piezoelectric response by the in-phase output channel of an external lock-in amplifier (SR830, Stanford Research Systems) with a typical sensitivity of $200 \mu \mathrm{V}$ and time constant of $10 \mathrm{~ms}$. Finally, we extract the area density of vortices from the PFM images.

We cool our samples at two rates that overlap with the fastest quenches performed in Ref. [45] -0.3 and $3 \mathrm{~K} / \mathrm{min}$ - to verify that we obtain a comparable density of defects in our experiments. The good agreement between the vortex densities formed in $\mathrm{ErMnO}_{3}$ (Ref. [45]) and those formed in $\mathrm{YMnO}_{3}$ [this work, two leftmost blue points in Fig. 4(a), domain structure shown in Fig. 4(b)] when quenched at the same rates confirms the independence of the vortex-core density from the choice of $R$. Subsequently, we quench the same samples at higher cooling rates of 195 and $1360 \mathrm{~K} / \mathrm{min}$ [rightmost blue points in Fig. 4(a)], resulting in the domain structures shown in Figs. 4(c) and 4(d), respectively. Surprisingly, an increase in the cooling rate leads to a lowering of the density of vortex cores and thus to larger domains. This behavior is opposite to that predicted by the standard Kibble-Zurek mechanism and may be described as "anti-Kibble-Zurek" behavior. The crossover between the two regimes occurs at a cooling rate of about $10 \mathrm{~K} / \mathrm{min}$.

This crossover point corresponds to a correlation length (and hence a crossover domain size) of about $1.1 \mu \mathrm{m}$ and a relaxation time of about $3.1 \times 10^{-3} \mathrm{~s}$, with a characteristic information transfer velocity of about $3.5 \times 10^{-4} \mathrm{~m} / \mathrm{s}$, considerably reduced from the speed of sound by the critical slowing down.

We emphasize that the observed behavior is highly reproducible: We have repeated our measurements on $\mathrm{YMnO}_{3}$ samples grown in different batches and have verified that there are no cumulative effects in consecutive annealing cycles. The error bars in Fig. 4(a) describe the variation of the vortex density when a sample from a different batch is used and when a data point for slow cooling rates is reproduced after taking the data point at the highest cooling rate. We therefore conclude that our results suggest an evolution out of the Kibble-Zurek regime at a cooling rate of about $10 \mathrm{~K} / \mathrm{min}$ in the hexagonal manganites.

\section{DISCUSSION: POSSIBLE ORIGINS OF THE CROSSOVER BETWEEN THE KIBBLE-ZUREK AND THE "ANTI-KIBBLE-ZUREK" REGIMES}

A number of possible deviations from Kibble-Zurek behavior have been discussed in the literature, but none of them is consistent with our measurements. Zurek [46] showed that vortex-antivortex annihilation becomes significant at fast quench rates where domains are smaller and topological defects are closer together. Such vortexantivortex annihilation causes a leveling off of the rate at which the density of vortex cores increases with cooling rate, but it does not cause the decrease in density that we observe. The effect of nonlinearity in the quench rate on the density of defects has been calculated to yield a modified scaling law [50], which again would not cause our observed turnaround at fast cooling rates. In addition, inhomogeneous cooling, and departures from linear cooling, which are both more likely in the fast-quench regime, have been shown to result in a suppression of defect formation [51,52] but again should result in a leveling off rather than our observed pronounced downturn. The authors of Ref. [45] suggested that the observed production of defect-antidefect pairs could be the result of a KosterlitzThouless transition [53], in which vortex-antivortex pairs are formed above the transition temperature and are annihilated as the system is cooled. As a result, more vortex cores survive during a fast quench when the pairs do not have time to be annihilated. This behavior is the opposite of our observed fast-quenching behavior. In addition, a Kosterlitz-Thouless system would show a dramatic change in the density of vortices after repeated annealing cycles, as well as a dependence on the temperature at which the quench begins, neither of which we observe in our experiment [54].

A possible extrinsic influence on the domain structure could be differences in chemical-defect concentration caused by the different cooling rates, such as offstoichiometry, antisite formation, or charge screening at the domain walls. To test for this possibility, we heat our samples to within $2 \%$ of the transition temperature, and anneal them at this temperature for six hours under the conditions described above. No changes in the domain structure have been observed on heating until the samples reach $1270 \mathrm{~K}$ (just below $T_{\mathrm{C}}$ ), when minor isolated domain-wall movements, and, once, the formation of a vortex-antivortex pair have been observed. On heating the samples to $1320 \mathrm{~K}$ (just above $T_{\mathrm{C}}$ ), we obtain a completely new, but statistically consistent domain pattern. 
These data and the aforementioned reproducibility of our data points in Fig. 4 suggest that chemical drift effects do not play a role.

We conclude, therefore, that our observed transition out of the Kibble-Zurek regime is likely intrinsic. One possibility for the origin of the transition is a breakdown of the Kibble-Zurek assumption that the system responds adiabatically as it cools from high temperature to the freeze-out temperature, which would in turn cause a breakdown of the scaling behavior. A second possibility is that ultrafast cooling causes the discrete sixfold symmetry of the crystal lattice to manifest at the transition temperature, so that the continuous symmetry of the 3D XY model, and in turn its scaling exponents, are no longer applicable. The behavior we observe in the fast-cooling regimeslower cooling leading to a larger number of smaller domains-is of course reminiscent of nucleationdominated behavior, with an activation energy for formation of the low-symmetry phase from the high-symmetry phase. Nucleation-dominated phase transitions show characteristic first-order behavior, and a longer time spent at the transition allows a larger number of smaller domains to nucleate. We note that, at the freeze-out temperature corresponding to the crossover quench rate, the order parameter for the trimerization, $\eta=\left(\frac{T-T_{\mathrm{C}}}{T_{\mathrm{C}}}\right)^{\beta}$, has already reached $0.5 \%$ of its saturation value, taking the experimental value of $\beta=0.27$ [44]. It is possible that this discontinuity is sufficient to induce a first-order response. An alternative scenario is the fluctuation-induced first-order behavior proposed for prototypical second-order phase transitions such as the normal-to-superconducting transition and for the nematic-smectic transition in liquid crystals [55], both of which belong to the same universality class - the 3D XY model - as the hexagonal manganites. With either origin, such an induced first-order transition could also explain the current controversy regarding the order of the trimerization transition in the hexagonal manganites, with most experiments showing second-order behavior, but occasional reports of first-order characteristics.

\section{SUMMARY}

We have shown that the multiferroic hexagonal manganites, $R \mathrm{MnO}_{3}$, are model systems for testing the KibbleZurek mechanism. Mathematically, they fulfill the symmetry requirements for the formation of topological defects, and, practically, the defects are readily detectable, the quench rate can be varied over a wide range of relevant time scales, and extrinsic factors that might influence the phase-transition behavior are absent. Our quantitative calculations of topological defect density as a function of cooling rate using the conventional Kibble-Zurek model and parameters obtained using density-functional theory, agree with literature data in the slow cooling limit where the conventional Kibble-Zurek mechanism is applicable. Our measurements of defect density at fast cooling rates, however, reveal a surprising, apparently "anti-KibbleZurek" behavior in which faster cooling yields lower defect densities, reminiscent of a nucleation-dominated phase transition.

\section{ACKNOWLEDGMENTS}

We thank Pierre Tolédano, Maxim Mostovoy, and Ali Mozaffari for invaluable discussions, Sang-Wook Cheong for sharing the data in Fig. 4(a) prior to its publication, and the Cosmic Superstrings Meeting (Portsmouth, United Kingdom, 2011) for allowing us to attend and discuss our ideas. This work was supported financially by the ETH Zürich, by the ERC Advanced Grant program, No. 291151, and by the Max Rössler Prize of the ETH Zürich. M.F. thanks the IMI Program of the National Science Foundation under Contract No. DMR-0843934, managed by the International Center for Materials Research, UC Santa Barbara, for sabbatical support. Calculations were performed at the Swiss Supercomputing Center (CSCS) and the Center for Scientific Computing at the CNSI and MRL: an NSF MRSEC (DMR-1121053) and NSF CNS0960316.

[1] M. Hindmarsh, C. Ringeval, and T. Suyama, $C M B$ Temperature Trispectrum of Cosmic Strings, Phys. Rev. D 81, 063505 (2010).

[2] A. Vilenkin and E.P.S. Shellard, Cosmic Strings and Other Topological Defects (Cambridge University Press, Cambridge, England, 1994).

[3] T. Vachaspati, Dark Strings, Phys. Rev. D 80, 063502 (2009).

[4] N. Bevis, M. Hindmarsh, M. Kunz, and J. Urrestilla, Fitting Cosmic Microwave Background Data with Cosmic Strings and Inflation, Phys. Rev. Lett. 100, 021301 (2008).

[5] R. Jeannerot, J. Rocher, and M. Sakellariadou, How Generic Is Cosmic String Formation in Supersymmetric Grand Unified Theories, Phys. Rev. D 68, 103514 (2003).

[6] T. W. B. Kibble, Topology of Cosmic Strings and Domains, J. Phys. A 9, 1387 (1976).

[7] W.H. Zurek, Cosmological Experiments in Superfluid Helium?, Nature (London) 317, 505 (1985).

[8] P.C. Hendry, N.S. Lawson, R.A.M. Lee, P. V.E. McClintock, and C.D.H. Williams, Generation of Defects in Superfluid ${ }^{4} \mathrm{He}$ as an Analogue of the Formation of Cosmic Strings, Nature (London) 368, 315 (1994).

[9] M.E. Dodd, P.C. Hendry, N.S. Lawson, P. V.E. McClintock, and C.D.H. Williams, Nonappearance of Vortices in Fast Mechanical Expansions of Liquid ${ }^{4} \mathrm{He}$ through the Lambda Transition, Phys. Rev. Lett. 81, 3703 (1998).

[10] R. J. Rivers, Slow ${ }^{4}$ He Quenches Produce Fuzzy, Transient Vortices, Phys. Rev. Lett. 84, 1248 (2000).

[11] C. Bauerle, Yu. M. Bunkov, S. N. Fisher, H. Godfrin, and G. R. Pickett, Laboratory Simulation of Cosmic String Formation in the Early Universe Using Superfluid ${ }^{3} \mathrm{He}$, Nature (London) 382, 332 (1996). 
[12] V. M. H. Ruutu, V. B. Eltsov, A. J. Gill, T. W. B. Kibble, M. Krusius, Yu. G. Makhlin, G. E. Placais, B. Volovik, and Wen $\mathrm{Xu}$, Vortex Formation in Neutron-Irradiated Superfluid ${ }^{3} \mathrm{He}$ as an Analogue of Cosmological Defect Formation, Nature (London) 382, 334 (1996).

[13] R. Monaco, J. Mygind, R. J. Rivers, and V. P. Koshelets, Spontaneous Fluxoid Formation in Superconducting Loops, Phys. Rev. B 80, 180501 (2009).

[14] C. N. Weiler, T. W. Neely, D. R. Scherer, A. S. Bradley, M. J. Davis, and B.P. Anderson, Spontaneous Vortices in the Formation of Bose-Einstein Condensates, Nature (London) 455, 948 (2008).

[15] A. Maniv, E. Polturak, and G. Koren, Observation of Magnetic Flux Generated Spontaneously during a Rapid Quench of Superconducting Films, Phys. Rev. Lett. 91, 197001 (2003).

[16] I. Chuang, R. Durrer, N. Turok, and B. Yurke, Cosmology in the Laboratory: Defect Dynamics in Liquid Crystals, Science 251, 1336 (1991).

[17] S. Ducci, P.L. Ramazza, W. Gonzalez-Vinas, and F. T. Arecchi, Order Parameter Fragmentation after a Symmetry-Breaking Transition, Phys. Rev. Lett. 83, 5210 (1999).

[18] B. B. van Aken, T. T. M. Palstra, A. Filippetti, and N. A. Spaldin, The Origin of Ferroelectricity in Magnetoelectric $\mathrm{YMnO}_{3}$, Nat. Mater. 3, 164 (2004).

[19] C. J. Fennie and K. M. Rabe, Ferroelectric Transition in $\mathrm{YMnO}_{3}$ from First Principles, Phys. Rev. B 72, 100103(R) (2005).

[20] M. Fiebig, Th. Lottermoser, D. Fröhlich, A. V. Goltsev, and R. V. Pisarev, Observation of Coupled Magnetic and Electric Domains, Nature (London) 419, 818 (2002).

[21] T. Lottermoser, T. Lonkai, U. Amann, D. Hohlwein, J. Ihringer, and M. Fiebig, Magnetic Phase Control by an Electric Field, Nature (London) 430, 541 (2004).

[22] T. Choi, Y. Horibe, H. T. Yi, Y. J. Choi, Weida Wu, and S.-W. Cheong, Insulating Interlocked Ferroelectric and Structural Antiphase Domain Walls in Multiferroic $\mathrm{YMnO}_{3}$, Nat. Mater. 9, 253 (2010).

[23] D. Meier, J. Seidel, A. Cano, K. Delaney, Y. Kumagai, M. Mostovoy, N. A. Spaldin, R. Ramesh, and M. Fiebig, Anisotropic Conductance at Improper Ferroelectric Domain Walls, Nat. Mater. 11, 284 (2012).

[24] H. L. Yakel, W. C. Koehler, E. F. Bertaut, and E. F. Forrat, On the Crystal Structure of the Manganese (III) Trioxides of the Heavy Lanthanides and Yttrium, Acta Crystallogr. 16, 957 (1963).

[25] T. Lonkai, D. G. Tomuta, U. Amann, J. Ihringer, R. W. A. Hendrikx, D. M. Tobbens, and J. A. Mydosh, Development of the High Temperature Phase of Hexagonal Manganites, Phys. Rev. B 69, 134108 (2004).

[26] T. Jungk, Á. Hoffmann, M. Fiebig, and E. Soergel, Electrostatic Topology of Ferroelectric Domains in $\mathrm{YMnO}_{3}$, Appl. Phys. Lett. 97, 012904 (2010).

[27] S. Artyukhin, K. T. Delaney, N. A. Spaldin, and M. Mostovoy, Landau Theory of Topological Defects in Multiferroic Hexagonal Manganites, arXiv:1204.4126v1.

[28] M. A. Kervaire and J. W. Milnor, Homotopy Groups of Spheres: I, Ann. Math. 77, 504 (1963).

[29] T.W. B. Kibble, in Topological Defects and the NonEquilibrium Dynamics of Symmetry Breaking Phase
Transitions, edited by Y.M. Bunkov and H. Godfrin, NATO Science Series C Vol. C549 (Kluwer Academic, Dordrecht, Netherlands, 2000), pp. 7-31.

[30] M. Hindmarsh and A. Rajantie, Defect Formation and Local Gauge Invariance, Phys. Rev. Lett. 85, 4660 (2000).

[31] T.W.B. Kibble, in Patterns of Symmetry Breaking: Proceedings of the Conference of the NATO Advanced Study Institute, Cracow, Poland, 2002, edited by H. Arodź, J. Dziarmaga, and W. H. Zurek, NATO Science Series II: Mathematics, Physics and Chemistry Vol. 127 (Springer, New York, 2003), pp. 3-36.

[32] A. I. Liechtenstein, V. I. Anisimov, and J. Zaanen, DensityFunctional Theory and Strong Interactions: Orbital Ordering in Mott-Hubbard Insulators, Phys. Rev. B 52, R5467 (1995).

[33] P.E. Blöchl, Projector Augmented-Wave Method, Phys. Rev. B 50, 17953 (1994).

[34] G. Kresse and J. Hafner, Ab Initio Molecular Dynamics for Liquid Metals, Phys. Rev. B 48, 13115 (1993).

[35] G. Kresse and J. Furthmüller, Efficient Iterative Schemes for Ab Initio Total-Energy Calculations Using a PlaneWave Basis Set, Phys. Rev. B 54, 11169 (1996).

[36] Q. H. Zhang, L. J. Wang, X. K. Wei, R. C. Yu, L. Gu, A. Hirata, M. W. Chen, C. Q. Jin, Y. Yao, Y. G. Wang, and X.F. Duan, Direct Observation of Interlocked Domain Walls in Hexagonal $R \mathrm{MnO}_{3}(R=\mathrm{Tm}, \mathrm{Lu})$, Phys. Rev. B 85, 020102(R) (2012).

[37] Y. Kumagai and N. A. Spaldin, Structural Domain Walls in Polar Hexagonal Manganites, arXiv:1207.4080.

[38] The ABINIT code is a common project of the Université Catholique de Louvain, Corning Incorporated, and other contributors [http://www.abinit.org].

[39] X. Gonze, J.-M. Beuken, R. Caracas, F. Detraux, M. Fuchs, G.-M. Rignanese, L. Sindic, M. Verstraete, G. Zerah, F. Jollet, M. Torrent, A. Roy, M. Mikami, Ph. Ghosez, J.-Y. Raty, and D.C. Allan, First-Principles Computation of Material Properties: The ABINIT Software Project, Comput. Mater. Sci. 25, 478 (2002).

[40] X. Gonze, B. Amadon, P.-M. Anglade, J.-M. Beuken, F. Bottin, P. Boulanger, F. Bruneval, D. Caliste, R. Caracas, M. Cote et al., ABINIT: First-Principles Approach of Materials and Nanosystem Properties, Comput. Phys. Commun. 180, 2582 (2009).

[41] A. Togo, F. Oba, and I. Tanaka, First-Principles Calculations of the Ferroelastic Transition between Rutile-Type and $\mathrm{CaCl}_{2}$-Type $\mathrm{SiO}_{2}$ at High Pressures, Phys. Rev. B 78, 134106 (2008).

[42] K. Parlinski, Z. Q. Li, and Y. Kawazoe, First-Principles Determination of the Soft Mode in Cubic $\mathrm{ZrO}_{2}$, Phys. Rev. Lett. 78, 4063 (1997).

[43] M. Campostrini, M. Hasenbusch, A. Pelissetto, and E. Vicari, Theoretical Estimates of the Critical Exponents of the Superfluid Transition in ${ }^{4} \mathrm{He}$ by Lattice Methods, Phys. Rev. B 74, 144506 (2006).

[44] A.S. Gibbs, K.S. Knight, and P. Lightfoot, HighTemperature Phase Transitions of Hexagonal $\mathrm{YMnO}_{3}$, Phys. Rev. B 83, 094111 (2011).

[45] S. C. Chae, N. Lee, Y. Horibe, M. Tanimura, S. Mori, B. Gao, S. Carr, and S.-W. Cheong, Direct Observation of the Proliferation of Ferroelectric Loop Domains and VortexAntivortex Pairs, Phys. Rev. Lett. 108, 167603 (2012). 
[46] A. Yates and W.H. Zurek, Vortex Formation in Two Dimensions: When Symmetry Breaks, How Big Are the Pieces?, Phys. Rev. Lett. 80, 5477 (1998).

[47] J. Dziarmaga, Dynamics of a Quantum Phase Transition and Relaxation to a Steady State, Adv. Phys. 59, 1063 (2010).

[48] A. H. Guth, Inflationary Universe: A Possible Solution to the Horizon and Flatness Problems, Phys. Rev. D 23, 347 (1981).

[49] A. A. Starobinsky, Dynamics of Phase Transition in the New Inflationary Universe Scenario and Generation of Perturbations, Phys. Lett. B 117, 175 (1982).

[50] S. Mondal, K. Sengupta, and D. Sen, Theory of Defect Production in Nonlinear Quench across a Quantum Critical Point, Phys. Rev. B 79, 045128 (2009).
[51] T.W.B. Kibble and G.E. Volovik, On Phase Ordering behind the Propagating Front of a Second-Order Transition, JETP Lett. 65, 102 (1997).

[52] J. Dziarmaga, P. Laguna, and W. H. Zurek, Symmetry Breaking with a Slant: Topological Defects after an Inhomogeneous Quench, Phys. Rev. Lett. 82, 4749 (1999).

[53] J.M. Kosterlitz and D. J. Thouless, Ordering, Metastability and Phase Transitions in Two-Dimensional Systems, J. Phys. C 6, 1181 (1973).

[54] D. Meier, M. Lilienblum, P. Becker, L. Bohaty, N. A. Spaldin, R. Ramesh, and M. Fiebig, Translation Domains in Multiferroics, Phase Transit. 86, 33 (2013).

[55] B. I. Halperin, T. C. Lubensky, and Shang-keng Ma, FirstOrder Phase Transitions in Superconductors and SmecticA Liquid Crystals, Phys. Rev. Lett. 32, 292 (1974). 